\title{
Mesures des fluctuations glaciaires dans les Alpes françaises Collecte des données et résultats
}

\section{Measurements of glacial fluctuations in the French Alps Collection of data and results}

\author{
L. Reynaud \\ Laboratoire de glaciologie et géophysique de l'environnement du CNRS, Grenoble \\ Annexe : Bilan du glacier de Sarennes (Saison 1982-83), par F. Valla, Division Nivologie, \\ CEMAGREF, Grenoble
}

Dès la fin du siècle dernier l'intérêt porté aux variations de glaciers a provoqué en France des campagnes de mesures sur de nombreux appareils. C'est ainsi que les glaciers ont commencé à être cartographiés et inventoriés et qu'ont débuté réellement les mesures de variations annuelles, suivant une méthodologie fixée par Vallot avec ses études sur la langue de la Mer de Glace de 1891 à 1899. En effet après avoir pratiqué le système de mesure en vogue en Europe à ce moment là qui consistait à mesurer non seulement l'avance ou le recul du glacier mais aussi l'altitude de profils fixes, avec la progression des lignes de pierres marquées, il s'est aperçu que pour pouvoir parler de variations de vitesse il fallait les mesurer chaque année au même lieu, en replaçant ces marques sur les profils origines.

Cette méthode a été reprise par l'administration des Eaux et Forêts dès 1907 pour quelques glaciers et surtout à partir de 1921 pour la majorité (Mougin, 1930).

Hormis quelques interruptions, comme celle due à la seconde guerre mondiale, les mesures ont été poursuivies jusqu'en 1966, époque du changement de statut des Eaux et Forêts.

A partir de ce moment là, il n'y a eu que quelques séries poursuivies dans le massif du Mt Blanc (Mer de Glace, Argentière et Tré la Tête) principalement pour les besoins d'Electricité de France.

Ce n'est qu'à partir de 1975 que le CEMAGREF a repris la surveillance des glaciers par photogrammétrie aérienne à période de 3 ans, qui doit permettre par restitution d'établir les fluctuations de longueur et de niveaux, mais, hélas, pas de vitesses.

Quel est le but de toutes ces mesures glaciologiques?

C'est évidemment de connaitre comment varient les glaciers au cours du temps et de remonter aux causes de ces variations qui sont les fluctuations climatiques.

Sur une année, l'ensemble des variations des précipitations et des températures modifie le régime d'alimentation du glacier, ce qui se traduit par des changements de forme, altitude, surface, de dynamique : débit, vitesse et finalement au bout de la chaine il y a la variation du front du glacier.

C'est donc par l'étude de la manifestation directe du climat sur le glacier : le bilan, qu'il faut commencer.

Le bilan de masse glaciaire

Malheureusement ces mesures sont récentes, elles n'ont débuté qu'en 1946 en Suède avec le Storglaciären (W. Shytt, 1980) et dans les Alpes le plus ancien glacier à être mesuré de façon directe est le Glacier de Sarennes depuis 1949 (F. Valla, 1984).

C'est pourquoi le Laboratoire de Glaciologie, dès sa création, a focalisé son activité sur l'étude des bilans en développant des méthodes de mesures tant en zone d'accumulation qu'en zone d'ablation et l'analyse spatio-temporelle de la répartition des bilans glaciaires.

Cette étude a conduit à la formulation du modèle linéaire statistique de variation du bilan (Lliboutry, 1974), à partir de 16 années et 32 sites de relevés dans la zone d'ablation de St-Sorlin.

Le bilan annuel l'année $t$ au site $j$ est :

$$
b_{j t}=\alpha_{j}+\beta_{t}+\varepsilon_{j t}
$$

$\alpha_{j}$ est un paramètre géographique indépendant de l'année, qu'on peut chercher à corréler avec l'altitude, l'exposition, la courbure de la surface, la proximité des bords du glacier, etc. pour une région donnée.

$\beta$, est un paramètre ne dépendant que de l'année et non pas du site, de moyenne nulle (= centré).

$\varepsilon_{i t}$ est un résidu aléatoire centré dépendant, plus que des erreurs de mesure, de fluctuations très locales sans intérêt pour le comportement d'ensemble du glacier. 
L'étude statistique montre qu'on peut considérer les $\varepsilon_{j}$ comme à peu près indépendants entre eux et ayant une même loi gaussienne de distribution (écart type : $0,20 \mathrm{~m}$ de glace).

La validité de ce modèle a été étendue à l'ensemble du Glacier de St-Sorlin (Vallon, Leiva, 1982) et la zone d'ablation de la Mer de Glace (Reynaud, 1977).

L'intérêt du modèle statistique linéaire est double : d'abord il permet une exploitation optimale de toutes les données dont on dispose. Il $\mathrm{y}$ a de très nombreux sites où les bilans manquent pour certaines années. Souvent, surtout vers la ligne d'équilibre, des balises d'ablation n'ont pas pu être retrouvées une année ou plusieurs années consécutives mais l'ont été une année ultérieure, si bien qu'on dispose en ces sites seulement de bilans cumulés pour plusieurs années consécutives.

Avec le modèle statistique linéaire il est possible d'utiliser aussi toutes ces mesures pour déterminer les " meilleurs estimateurs linéaires sans biais " des $\alpha_{j}$ et des $\beta$.

Ensuite le modèle permet de limiter la mesure des bilans annuels à quelques zones d'ablation peu inclinées, non crevassées ni avalancheuses, d'accès facile, où l'on surveillera tous les ans un réseau dense de balises, de façon à déterminer les $\beta_{i}$.

C'est finalement ce que tentaient de faire les services des Eaux et Forêts en déterminant chaque année les positions des fronts et le niveau de quelques profils transversaux. Un essai de détermination de l'ablation à partir de ces mesures de surface avait été fait par Vallot. Il trouvait quelques $4 \mathrm{~m}$ d'ablation dans une zone où elle est voisine de $12 \mathrm{~m}$ ! En fait pour faire le bilan d'ablation d'un tronçon de glacier compris entre les deux sections droites passant par les profils, il faut aussi connaître la topographie du lit. Dans ce cas la conservation du flux écrite par l'équation de continuité, permet d'obtenir le bilan, pour tout le laps de temps où les variations d'altitude et de vitesse sont connues.

C'est ce qui a pu être fait pour le Glacier de Gébroulaz de 1908 à 1950 (Reynaud, Vallon et Carle, 1983). La série de 43 ans ainsi obtenue est portée sur la figure 1 en même temps que celle reconstituée pour le Glacier de Sarennes (Martin, 1977) et celle mesurée par la méthode hydrologique sur le glacier d'Aleth (Kasser, Aellen, 1980). L'intérêt de ces séries obtenues par l'équation de continuité est fondamental à plusieurs titres. Tout d'abord elles étendent notre connaissance du bilan sur de longues périodes de temps et permettent de tester les reconstitutions de bilans obtenues à partir des paramètres météorologiques comme celle de Martin.

D'autre part, grâce à l'héritage des mesures des Eaux et Forêts, quelques autres glaciers sont susceptibles de fournir d'aussi longues séries une fois la topographie du lit déterminée par sondages (forages, sismique ou radar). C'est-à-dire qu'on va être en mesure de décrire la répartition du bilan de masse glaciaire dans l'espace et le temps et tester l'extension du modèle linéaire d'une façon beaucoup plus solide qu'on a pu le faire jusqu'ici.

Enfin au point de vue méthodologique, la méthode de l'équation de continuité fournit une alternative intéressante à la détermination directe de bilan en réduisant les opérations de terrain. Dans cette optique les glaciers de Gébroulaz, Blanc et Noir ont été rééquipés en 1983 du réseau de mesures utilisé autrefois par les Eaux et Forêts avec deux profils et marquage de pierres peintes.

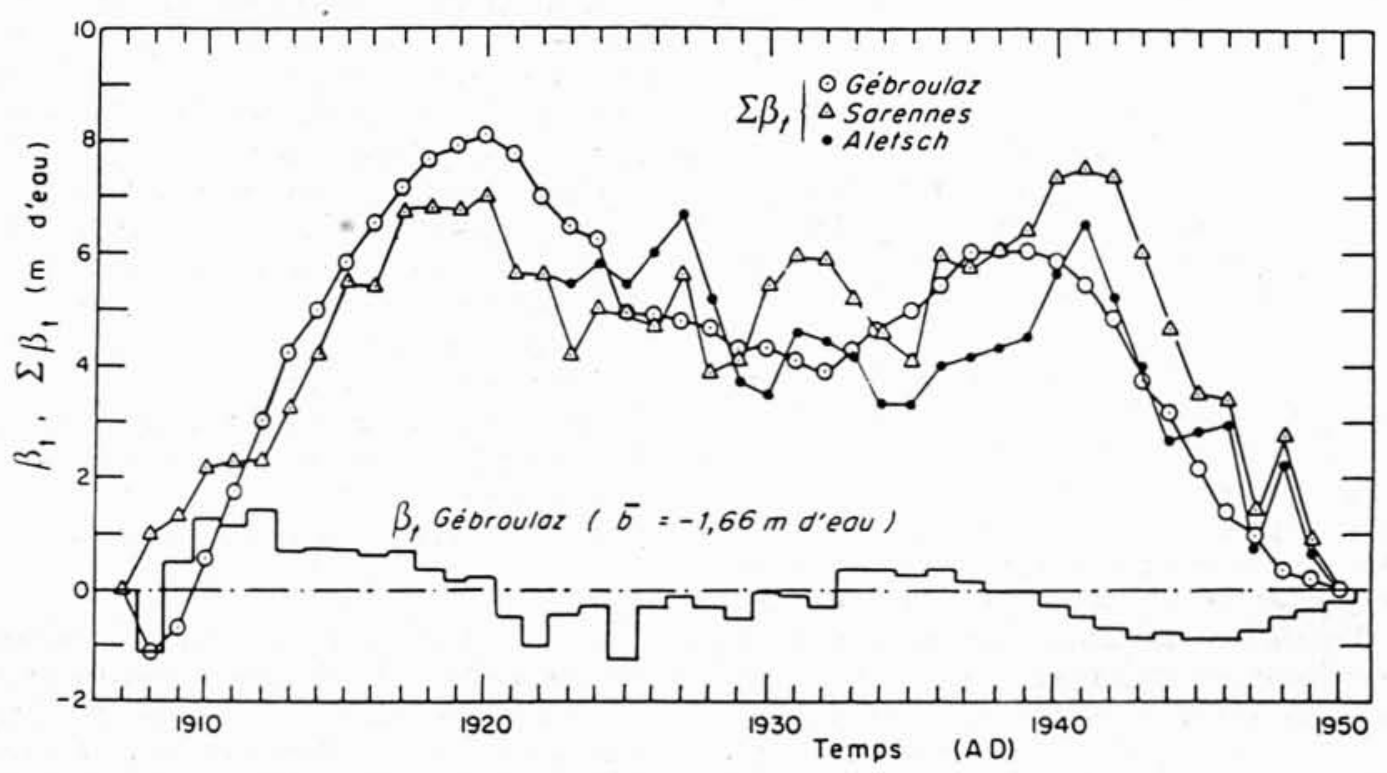

Figure 1 - Variation du bilan $\beta$, entre les deux profils du Glacier de Gébroulaz et comparaison de ces variations à celles reconstituées pour le Glacier de Sarennes (Martin, 1977) et à celles mesurées sur le Glacier d'Aletsch (Kasser et Allen, 1980). 
Le réseau d'observation des glaciers français en 1983

Il comprend des glaciers des massifs du Mont-Blanc, de la Vanoise, des Grandes Rousses, des Ecrins et des Pyrénées. Les glaciers qui bénéficient de mesures annuelles sont répertoriés dans le tableau $I$ avec les principaux renseignements concernant les mesures effectuées et les séries temporelles disponibles. Il faut ajouter à cet échantillon les mesures photogrammétriques effectuées par le CEMAGREF depuis 1975 (R. Burnet, 1984).

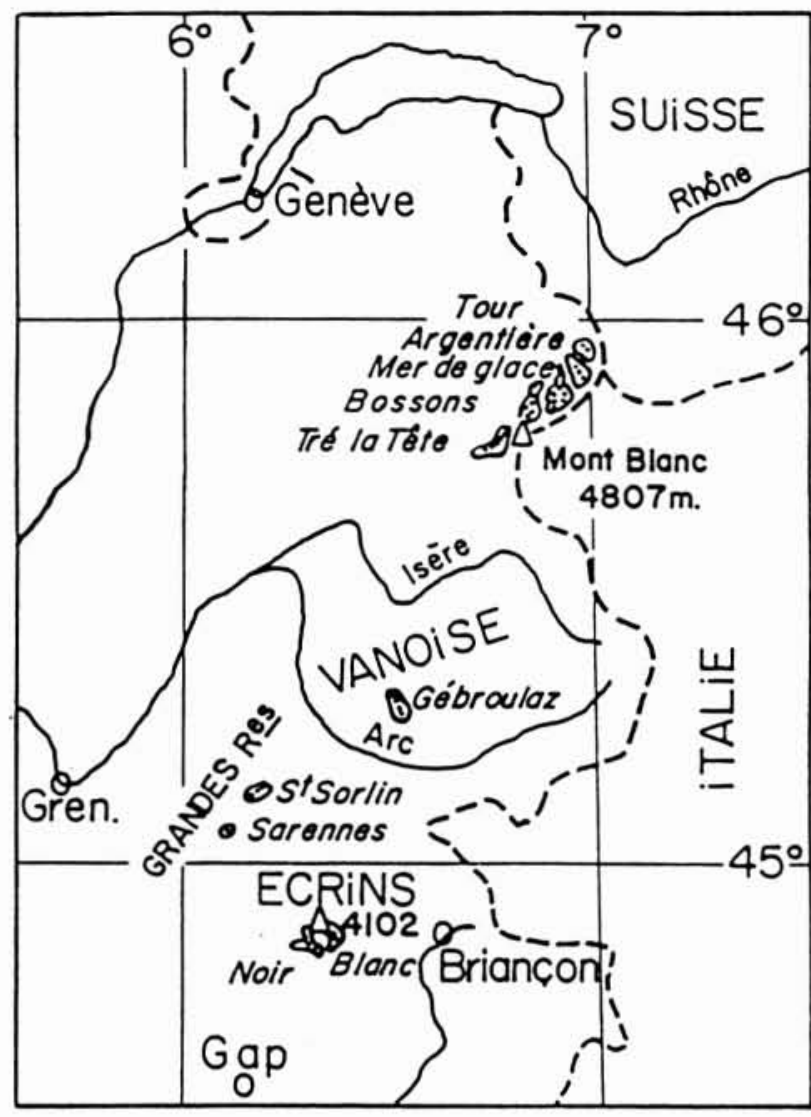

Figure 2 - Carte de situation des glaciers suivis annuellement dans les Alpes Françaises.

\begin{tabular}{|c|c|c|c|c|c|c|c|c|}
\hline Massif & Glaciers & Front & Niveaux & Vitesses & Bilans & Photogrammétrie & Cartes & Observateurs \\
\hline $\begin{array}{l}\text { Mont- } \\
\text { Blanc }\end{array}$ & $\begin{array}{c}\text { Tour } \\
\text { Argentière } \\
\text { Mer de } \\
\text { Glace } \\
\text { Bossons } \\
\text { Tré-la-Tête }\end{array}$ & $1870 \ldots$ & $1891 \ldots 1983$ & $\begin{array}{r}\text { Vallot : } 1891-99 \\
\text { E \& F : } 1912-60 \\
\text { Glacio : } 1968-74 \\
1978 \ldots\end{array}$ & \begin{tabular}{|c|}
$1975 \ldots$ \\
$1968-75$ \\
$1978 \ldots$ \\
$1973-74$ \\
$1982 \ldots$
\end{tabular} & $\begin{array}{ll} & 1939 \\
& 1949 \\
& 1952 \text { (CAF) } \\
\text { I.G.N. } & 1958 \\
& 1967 \\
& 1970 \\
& \\
& 1974 \\
& \\
\text { CEMAGREF : } 76,79,82 \\
\text { Glaciologie : Bossons } \\
\text { 1966 }\end{array}$ & \begin{tabular}{|c|} 
restitutions \\
diverses \\
1958 \\
1967
\end{tabular} & $\begin{array}{l}\text { E \& F, } \\
\text { EDF + Glaciologie } \\
\text { E \& F, } \\
\text { Mogtangers } \\
\text { E \& F, EDF } \\
\text { EDF + Glaciologie }\end{array}$ \\
\hline Vanoise & Gébroulaz & $\begin{array}{r}1730 \\
1860 \\
1907 \ldots\end{array}$ & $\begin{array}{l}\text { E\&F 1907-66 } \\
\text { Glacio } 1983 . .\end{array}$ & $\begin{array}{l}\text { E\&F : } 1907-66 \\
\text { Glacio : } 1983 \ldots\end{array}$ & $\begin{array}{c}1908-50 \\
1978 \ldots\end{array}$ & $\begin{array}{l}\text { IGN : } 1952,1967, \\
\text { CEMAGREF : } 1977,80,83\end{array}$ & 1967 & $\begin{array}{l}\text { E \& F } \\
\text { Glaciologie - Parc }\end{array}$ \\
\hline $\begin{array}{l}\text { Grandes } \\
\text { Rousses }\end{array}$ & $\begin{array}{c}\text { Sarennes } \\
\text { Saint-Sorlin }\end{array}$ & 1905 & $\begin{array}{l}\text { balises } \\
\text { glacio. } \\
1956\end{array}$ & id. & $\begin{array}{c}1949 \ldots \\
1956 \ldots\end{array}$ & $\begin{array}{l}\text { CEMAGREF : 1975, 1978, } \\
81: \text { restituee } \\
\text { IGN : } 1927,67 \\
\text { CEMAGREF : } 1984\end{array}$ & \begin{tabular}{|c|}
1904,27 \\
59,67 \\
$1904,27,67$
\end{tabular} & $\begin{array}{l}\text { CEMAGREF } \\
\text { Glaciologie }\end{array}$ \\
\hline Ecrins & $\begin{array}{l}\text { Blanc } \\
\text { Noir }\end{array}$ & \begin{tabular}{|c|}
1815 \\
$1921-66$ \\
$1983 \ldots$ \\
\end{tabular} & $\begin{array}{c}1904 \\
1921-66 \\
1983 \ldots\end{array}$ & $\begin{array}{c}1921-1966 \\
1983 \ldots\end{array}$ & \begin{tabular}{|c|}
$1921-50$ \\
$1978 \ldots$ \\
$1983 \ldots$ \\
\end{tabular} & $\begin{array}{l}\text { IGN : } 1952,67,80 \\
\text { CEMAGREF : } 1975,78,81\end{array}$ & $\begin{array}{l}1905 \\
1921 \\
1967\end{array}$ & $\begin{array}{l}\text { E \& F } \\
\text { Glaciologie - Parc }\end{array}$ \\
\hline Pyrénées & $\begin{array}{l}\text { Taillon } \\
\text { Ossoue }\end{array}$ & & & & $1983 \ldots$ & $\begin{array}{l}\text { IGN } \\
\text { Univ. Pau + Alat : } \\
\text { J.P. Tihay }\end{array}$ & & $\begin{array}{l}\text { Université de Pau } \\
\text { + Parc Pyrénées } \\
\text { + CEMAGREF } \\
\text { + Glaciologie }\end{array}$ \\
\hline
\end{tabular}




\section{La variation de longueur}

Au point de vue des variations de longueur des glaciers, on dispose d'une intéressante série de comparaison fournie par 4 glaciers situés sur le même versant nord du Mont-Blanc : Bossons, Argentière, Trient et Mer de Glace.

La figure 3, où sont portées leurs variations depuis 1870 , montre des avancées semblables en 1890, 1920 et 1970, entrecoupées de reculs, dont celui de la décennie 40 à 50 constitue une caractéristique commune qu'on retrouve sur tous les autres glaciers de la chaîne des Alpes. En fait, cette simultanéité de variations n'est pas aussi nette : on constate en effet que les maximums sont décalés dans le temps, particulièrement lors de la dernière crue.

Les reprises d'avancée des différents glaciers lors de ces trois crues donnent sur les courbes des coudes plus nets qui permettent l'analyse graphique de la partie basse de la figure 3. Il apparait alors qu'à chaque crue les différents glaciers réagissent avec les mêmes temps de retard : c'est celui des Bossons qui réagit en premier, suivi de ceux d'Argentière et Trient, 4 à 7 ans plus tard, puis finalement vient la Mer de Glace avec 11 à 15 ans de retard.

Que se passe-t'il tout le long du glacier lorsque se prépare une avancée ou un recul du front ?

Pour l'analyser on dispose de relevés d'altitudes effectués en 5 profils en travers sur la zone d'ablation de la Mer de Glace. Pour la période 1957-1981, qui recouvre la fin de la grande décrue des années 40 à 50 et le début de la crue actuelle (fig. 4), on constate que la reprise d'épaississement s'est étagée dans le temps pour les profils depuis Trélaporte en 1965 jusqu'aux Mottets en 1972. Ensuite, alors que les 4 profils du bas continuent d'augmenter, le profil du haut, Tacul, établi en 1970, ne cesse de décroître. Le mécanisme de fluctuation de niveau le long du glacier n'est donc pas une simple variation homogène, mais correspond plutôt à la propagation d'un excès de masse. En prenant comme début de passage les instants repérés par une flèche sur le graphique, on obtient une vitesse de propagation de $500 \mathrm{~m}$ par an, ce qui est 5 fois la vitesse moyenne dans cette partie du glacier. Cette onde de crue représente une hauteur maximale de 5 à $6 \mathrm{~m}$, elle s'étale sur $7 \mathrm{~km}$. Des mesures semblables, bien que moins continues dans le temps, révèlent le même mécanisme sur les glaciers d'Argentière et des Bossons.
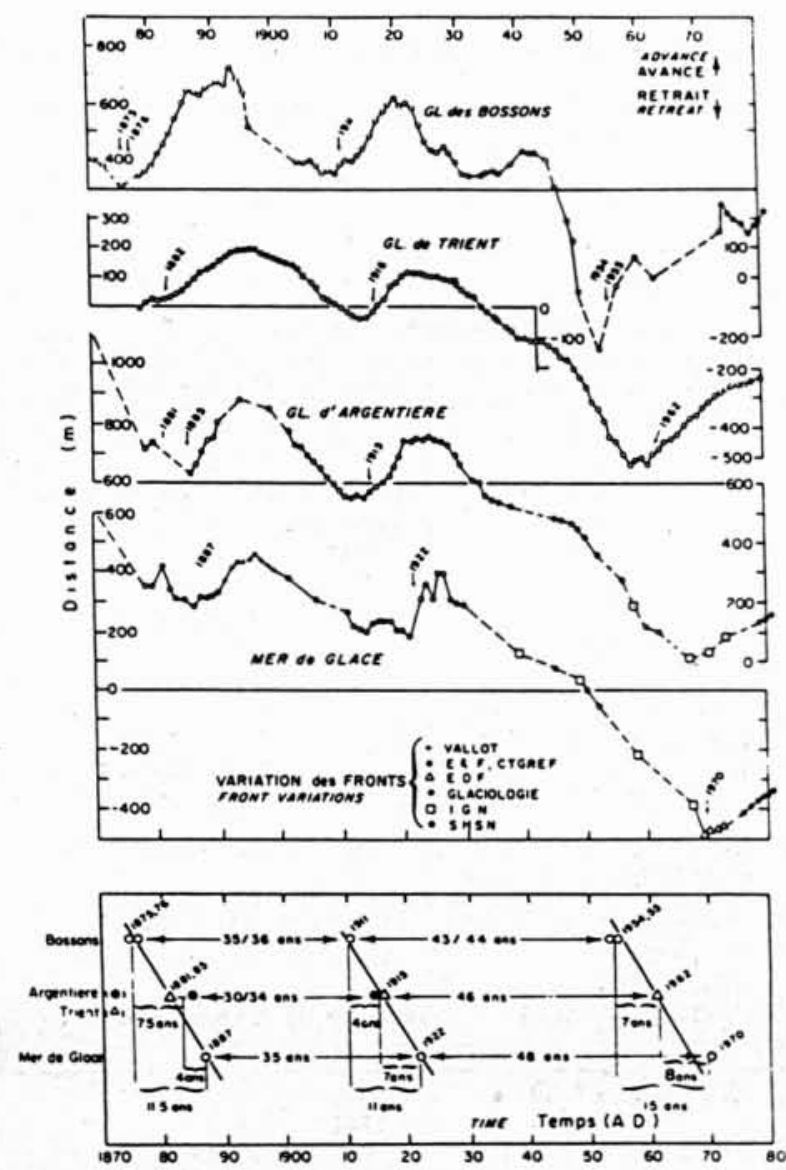

Figure 3 - Variations de longueur de 4 glaciers situés sur le même versant nord du Mont-Blanc de 1870 à 1981 et analyse graphique de leur temps de réponse lors des 3 crues principales.

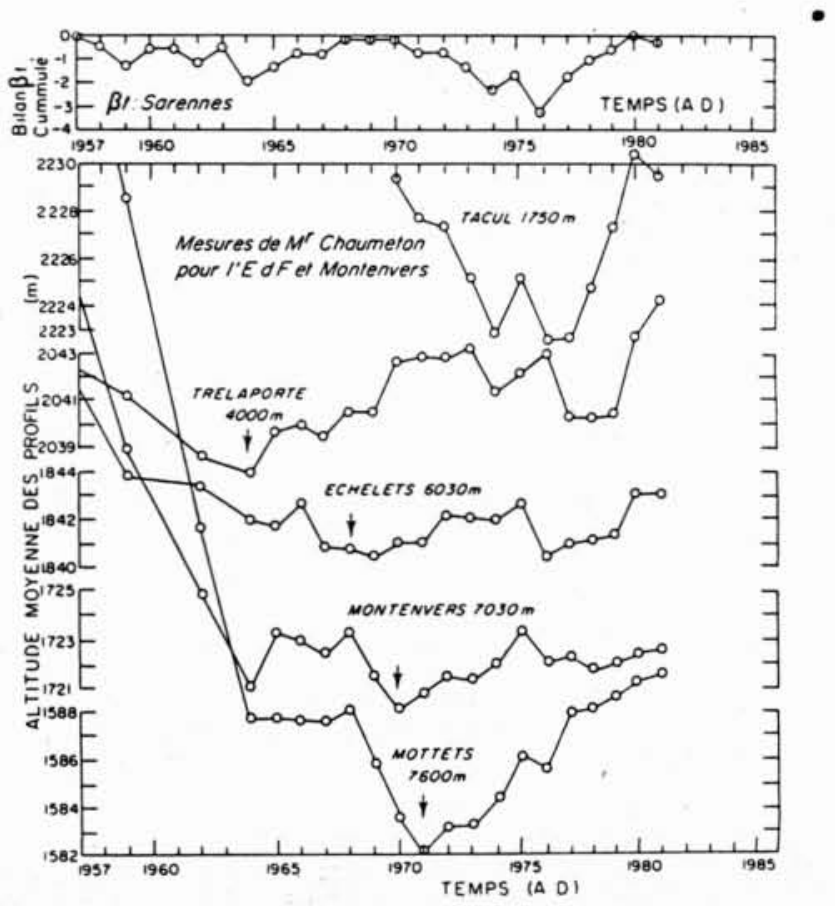

Figure 4 - Variation de l'altitude moyenne des profils transversaux relevés sur la Mer de Glace de 1957 à 1981. 


\section{Les variations de vitesses}

Pour décrire la dynamique de la Mer de Glace, on ne dispose pas de renseignements aussi continus que pour les fronts ou les niveaux, mais seulement de trois séries de mesures de vitesses qui sont :

- celles de Vallot de 1891 à 1899 avec des pierres marquées suivant le mouvement du glacier;
- des mesures EdF de 1912 à 1962 aux profils fixes et des valeurs données par le réseau longitudinal de balises suivi par le Laboratoire de Glaciologie de 1968 à 1974 (fig. 5).

Ces trois séries ne donnent pas les variations de vitesses de la même façon et on ne peut pas les relier facilement aux autres variations enregistrées de façon presque continue sur ce dernier siècle. Cependant en tirant profit du marquage annuel du Glacier par les bandes de Forbes et des caractéristiques des variations de vitesses tout le long
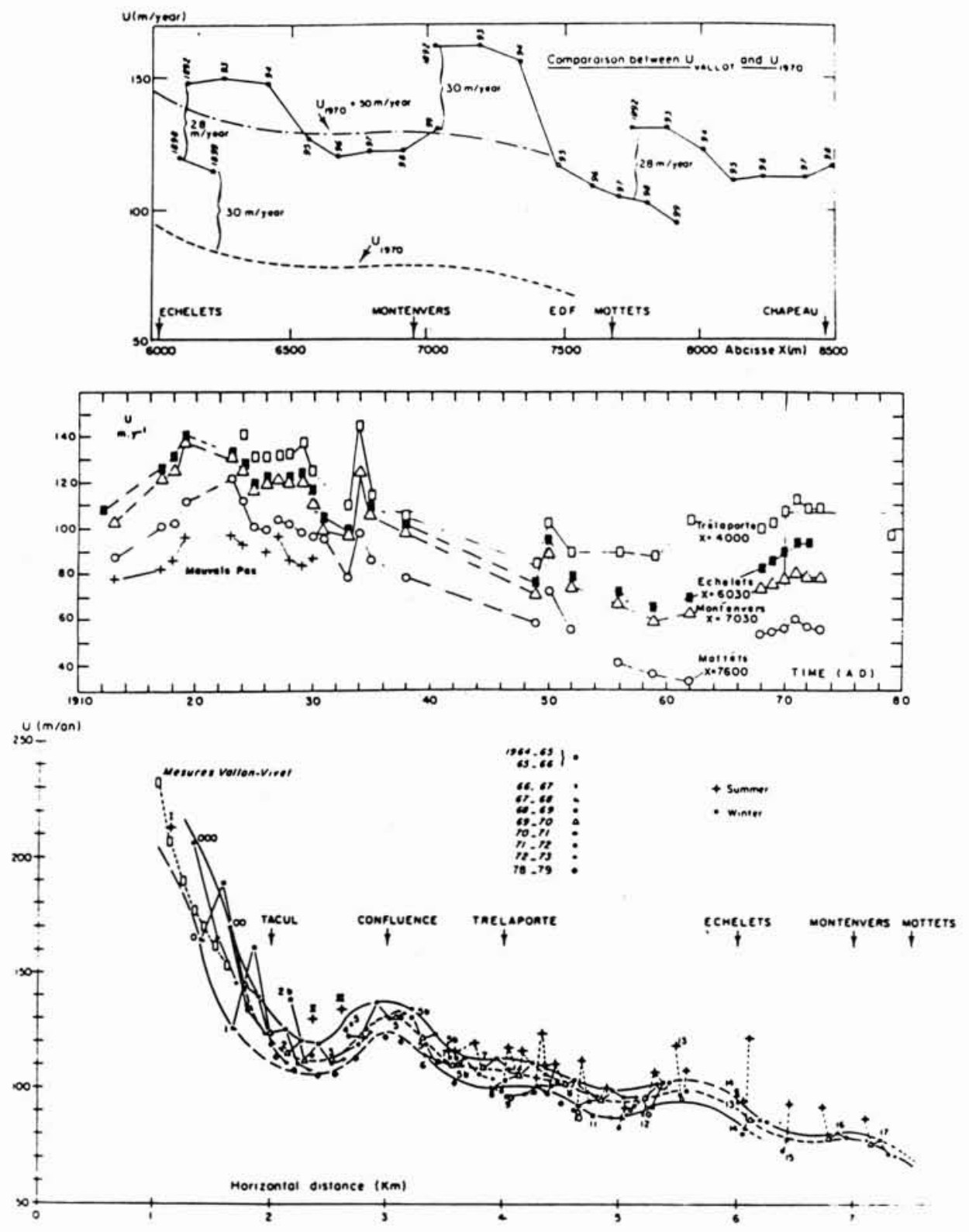

Figure 5 - Mesure des vitesses annuelles sur la Mer de Glace :

a) par Vallot avec trois lignes transversales de pierres peintes

suivant le mouvement de la glace,

b) par les Eaux-et-Forêts de 1912 à 1962 et par le Laboratoire de

Glaciologie de 1968 à 1973 et depuis 1978,

c) par le Laboratoire de Glaciologie avec une ligne de balises longitudinales. 
du glacier (fig. 5c) qui apparaissent synchrones pour les $6 \mathrm{~km}$ du glacier, on a pu reconstituer la série complète des variations de vitesses depuis 1888 . Pour cela on a utilisé pratiquement toutes les données disponibles en photogrammétrie aérienne ( 7 couvertures) de façon à obtenir des séries de valeurs indépendantes (fig. 6). Cette reconstitution rend bien compte des 3 séries d'observations et montre que les vitesses sont contrôlées par la partie supérieure du glacier, ici le glacier du Tacul, et qu'elles ne sont pas modifiées par le passage d'une onde de crue, comme cela se vérifie aux trois situations sur le siècle
(Lliboutry et Reynaud, 1981).

Ce même mécanisme de variation de vitesse se rencontre aussi sur le glacier d'Argentière où là la zone qui contrôle la vitesse est constituée par l'ensemble du plateau supérieur. Bien qu'on en ait qu'une description assez courte dans le temps, on remarque sur la figure 7 une variation quasi synchrome dans les 3 profils supérieurs d'une dizaine de mètres d'altitude seulement alors que les variations des profils situés au-dessous de la chute présentent des amplitudes beaucoup plus importantes.

Récemment, de 1976 à 1982 , on a vu la vitesse sur le

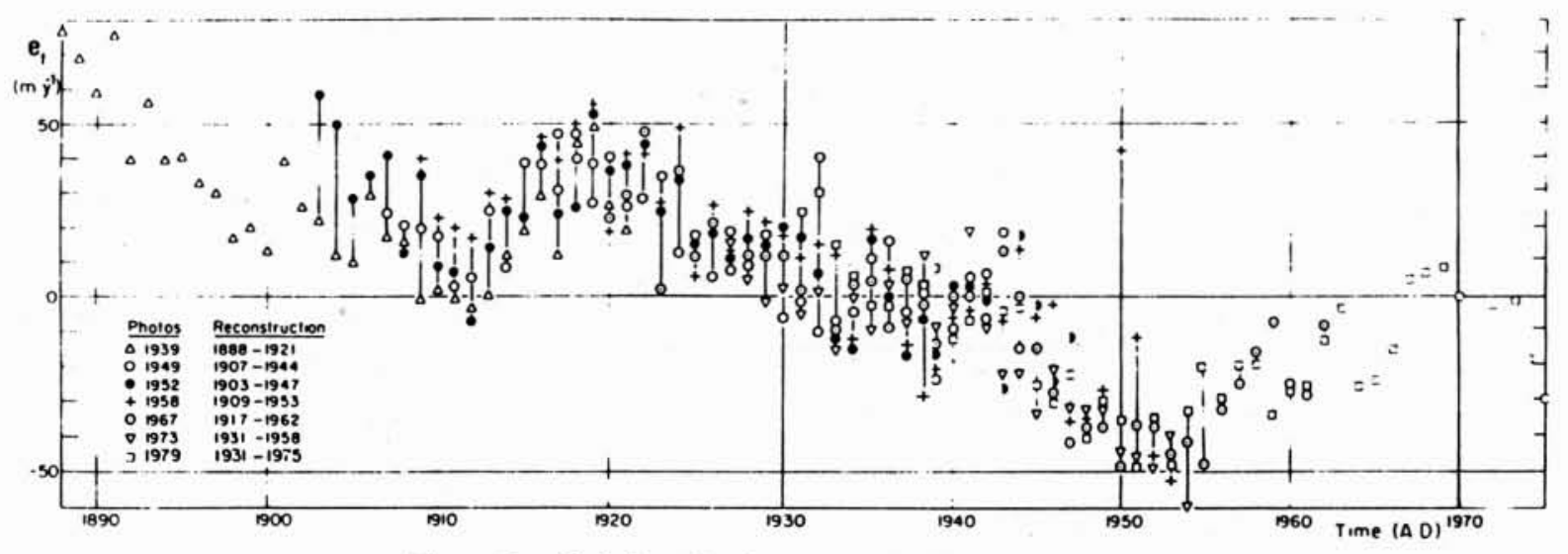

Figure 6 - Variations de vitesses par rapport à l'état de 1970 sur la Mer de Glace données par les différences de trajets des bandes de Forbes à partir de 7 restitutions photogrammétriques de l'IGN et du CEMAGREF.

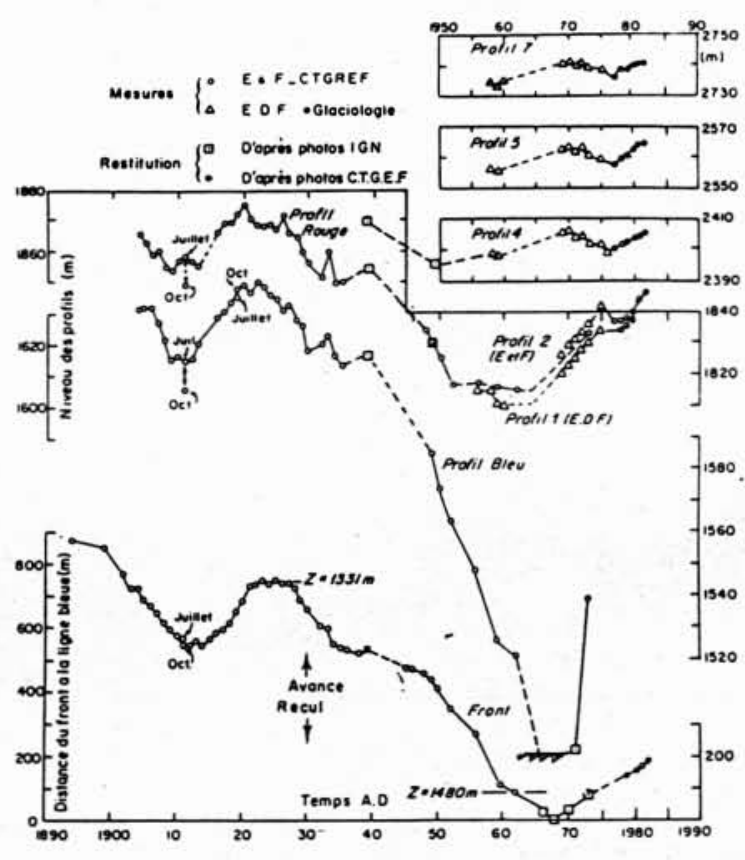

Figure 7 - Glacier d'Argentière : variation des niveaux et des fronts en fonction du temps.

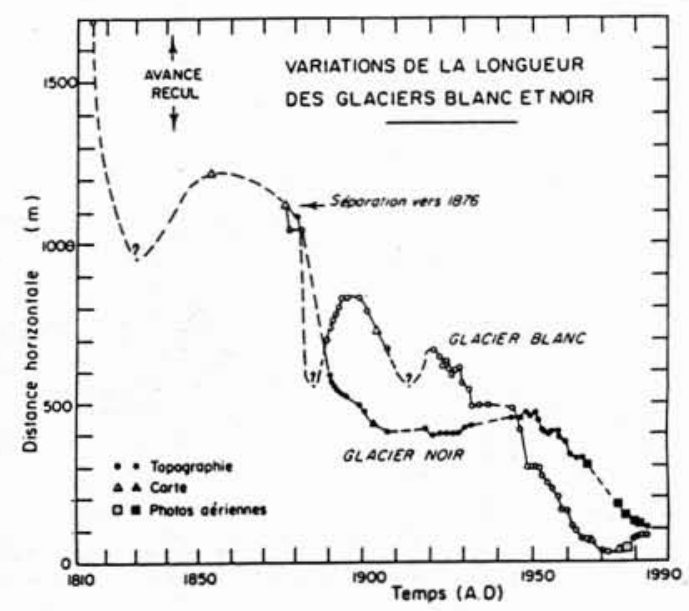

Figure 8 - Variations de longueur des glaciers Blanc et Noir depuis 1815 . 
plateau supérieur augmenter de $30 \%$, alors que l'altitude ne varie pendant le temps que de $5 \mathrm{~m}$ environ, c'est-à-dire $2 \%$ de l'épaisseur seulement.

Ces données illustrent clairement le rôle de ces zones de contrôle de fortes épaisseurs, dont l'altitude au cours du temps ne varie que très peu autour d'une position d'équilibre. Une fois cet équilibre d'écoulement rompu, soit par une surcharge ou un déficit, il y a accélération ou déccélération et tout l'écoulement de la langue à l'avảl est conditionné par ces modifications (Rapport à Emosson SA, 1983).

Dans le Massif des Ecrins, 2 glaciers voisins ont été suivis au cours du temps : les Glaciers Blanc et Noir. Ils présentent la particularité de varier en longueur depuis 1860 de façon très dissemblable (fig. 8). Alors que le Glacier Blanc présente les mêmes événements que ceux du Mt-Blanc (Letreguilly, 1984) le Glacier Noir ne montre pratiquement pas de changement en 1890, il se stabilise vers 1910 pour avancer régulièrement jusqu'à 1950. Ce n'est qu'à partir de ce moment là qu'il commence à reculer fortement jusqu'en 1983.

A cause de leur proximité ces deux glaciers doivent subir des conditions d'alimentation très voisines et ce qui fait la différence c'est la couverture morainique, inexistante sur le Glacier Blanc et régulière sur la langue du Glacier Noir (30 à $40 \mathrm{~cm}$ ). On doit avoir là une occasion favorable d'étude de l'effet de la couverture morainique sur le bilan du Glacier, et d'en tirer des renseignements sur le comportement des langues glaciaires toujours plus ou moins recouvertes.

C'est pourquoi nous avons effectué en août 1983 une prospection sismique entre les 2 profils Eaux et Forêts de façon à établir la série de bilan de masse de 1923 à 1966. Les premiers calculs montrent que le bilan moyen sur cette zone est de 2 mètres de glace/an, inférieur à celui du glacier Blanc pour une même altitude.

\section{Conclusion}

L'ensemble des mesures disponibles sur les glaciers français permet d'établir quelques longues séries pour les différents paramètres qui décrivent les variations des glaciers. En leur ajoutant quelques déterminations plus spécifiques telles que les sondages sismiques, ou en recoupant ces mesures d'une façon différente telle que les lignes longitudinales de balises on a pu dégager quelques modèles d'évolution et tester leur représentativité sur de longues périodes ( 50 à 100 ans).

Il est probable que la poursuite des analyses de cette base de données va nous permettre de préciser ces modèles et d'en découvrir d'autres.

Pour cela il est absolument nécessaire que les mesures en cours soient poursuivies chaque année, d'autant plus que la période actuelle présente un intérêt particulier avec la reprise d'avancée de la majorité des glaciers: les variations recueillies à ce moment là sont capitales pour la compréhension des mécanismes de variation.

\section{Références}

BURNET R., 1984. - Relevés aériens triennaux de l'état d'avancement ou de recul de certains glaciers, SHF. Paris, mars 1984.

CEMAGREF. - Archives des mesures glaciologiques des Eaux-et-Forêts et Photothèque, Saint-Martin d'Hères 38.

KASSER P. and AELLEN M., 1982. - Rapport annuel de la Commission Suisse des Glaciers. S.H.F., section de glaciologie, mars 1982, Paris.

LETREGUILLY A., 1982. - Analyse des mesures effectuées sur le Glacier Blanc, massif des Ecrins, France. DEA, sept. 1982, Université de Grenoble I, Laboratoire de Glaciologie du CNRS.

LLIBOUTRY L., 1974. - Multivariate statistical analysis of glacier annual balances. Journal of Glaciology, 13, n०69, p. 317-392.

LLIBOUTRY L., and REYNAUD L., 1981. - Global dynamics of a temperate valley glacier, Mer de Glace, and past velocities deduced from Forbes'bands. Journal of Glaciology, 27, no 96, p. 207-226.

MARTIN S., 1974. - Corrélations bilans de masse annuels facteurs météorologiques dans les Grandes-Rousses. Zeitschrift für Gletscherkunde und Glazialgeologie, 10, no 1-2, p. 89-100.

MARTIN S., 1977. - Analyse et reconstitution de la série de bilans annuels du glacier de Sarennes, sa relation avec les fluctuations du niveau de trois glaciers du massif du MontBlanc (Bossons, Argentière, Mer de Glace). Zeitschrift für Gletscherkunde und Glazialgeologie, 13, n०1-2, p. 127-153.

MOUGIN P., 1934. - Etudes glaciologiques, Tome 7, Paris, Direction des Eaux et du Génie Rural, Imprimerie Nationale.

REYNAUD L., 1977. - Glaciers fluctuations in the Mont-Blanc Area (French Alps). Zeitschrift für Gletscherkunde und Glazialgeologie, 13, n०1-2, p. 155-166.

REYNAUD L., 1980. - Can the linear balance model be extended to the whole Alps. World Glacier inventory, Riederalp workshop, sept. 1978. IAHS Bulletin, no 125, p. 273-284.

REYNAUD L., 1981. - Reconstitution of past velocities of Mer de Glace using Forbes'bands. Zeitschrift für Gletscherkunde und Glazialgeologie, 15, p. 149-163.

REYNAUD L., VALLON M. et CARLE C., 1983. - Analyse et synthèse des mesures glaciologiques effectuées sur le Glacier de Gébroulaz, Massif de la Vanoise, France. Travaux Scientifiques du Parc National de la Vanoise, 13, p. 9-29.

SCHYTT V., 1980. - The net mass balance of Storglaciären, Kebnekaise, Sweden related to the height of equilibrium line and to the height of the $500 \mathrm{mb}$ surface. Geografiska Annaler, $63 A$, no 3-4, p. 219-223.

VALLA F., 1984. - Bilan de masse du Glacier de Sarennes en 1983. S.H.F., Paris, mars 1984.

VALLON M., LEIVA J.-C., 1982. - Bilan de masse et fluctuations récentes du Glacier de Saint-Sorlin, Alpes Françaises. Zeitschrift für Gletscherkunde und Glazialgeologie, 17, no 1-2, p. 143-167. 


\section{Annexe : Bilan du glacier de Sarennes (année 1982-83), $35^{\mathrm{e}}$ année d'observations, par F. Valla, Division Nivologie, CEMAGREF, Grenoble}

\section{Données climatiques}

Le cycle 1982-1983, présente, tant pour les températures que pour les précipitations, des caractéristiques au-dessus de la moyenne des 35 années de mesures (1० de température et $120 \mathrm{~mm}$ de précipitation).

1. La période d'accumulation - octobre 1982 - mai 1983 - se caractérise par :

- un automne pluvieux (359 mm pour 259 de normale, soit $39 \%$ d'excédent) et doux (principalement en novembre et décembre) (température moyenne supérieure de plus de 10 ).

- un hiver normalement arrosé avec un mois de février très froid (1०8 au lieu de $3 \circ 9)$, janvier ayant été plus doux que la normale $(2 \circ 7$ au lieu $1 \circ 9)$.

- un printemps très maussade, peu ensolleillé et très humide (précipitations avril, mai de $122 \mathrm{~mm}+174 \mathrm{~mm}=296$ pour une valeur normale de 151 , soit un excédent de $96 \%$. Il restera gravé dans la mémoire des randonneurs de ski de printemps, qui en deux mois, n'eurent que quelques jours de beau temps.

2. La période d'ablation - juin 1983 - septembre 1983 - se caractérise par :

- des précipitations très inférieures à la normale (212 mm d'eau pour $346,2 \mathrm{~mm}$ de valeur moyenne, soit $39 \%$ de déficit). A remarquer que l'excédent d'avril-mai compense le déficit juin-septembre.

- des températures exceptionnellement élevées (juin $19 \circ 7 / 17 \circ 9$, juillet $24 \circ 4 / 20^{\circ} 3$, août $21^{\circ} 2 / 19 \circ 8$, septembre $18^{\circ} 2 / 16^{\circ} 9$ ), soit une température moyenne d'été de plus de $2^{\circ}$ supérieure à la normale.

Compte tenu de ces données climatiques, on peut s'attendre à une bonne accumulation, suivie d'une ablation continue et régulière, de fortes valeurs. D'où un bilan voisin de 0 et un très fort régime.

\section{Mesures}

Cinq reconnaissances (fig. 4) ont été faites du $1^{\text {et }}$ juin au 30 septembre. A partir de mi-septembre, le centre du glacier se déneige. Comme en septembre 81 et 82 , la glace vive apparait aux stations 2,3 et 4 .

\section{Caractéristiques du $35^{\circ}$ cycle $1982 / 83$}

L'analyse des valeurs de ce $35^{\mathrm{e}}$ cycle, ( fig. 5), montre :

- une accumulation exceptionnelle de 2,85 mètres, voisine du record $77(2 \mathrm{~m} \mathrm{78})$ provoquée par les très fortes chutes de neige d'avril et mai $\left(1^{\text {er }}\right.$ rang $127 \mathrm{~cm}$ d'écart par rapport à la moyenne de $\left.158 \mathrm{~cm}\right)$;

- une forte ablation, de $275 \mathrm{~m}$ de valeur en eau. C'est le $4^{e}$ rang (+ 69 d'écart par rapport à la moyenne de 206);

- un bon bilan positif de $+10 \mathrm{~cm}$ pour $-48 \mathrm{~cm}$ de moyenne sur les 35 cycles $(+58 \mathrm{~cm}$ par rapport au bilan centré) c'est le $10^{c}$ rang et l'ensemble des 7 cycles successivement positif $(1976 / 77 / 1982 / 83)$ a énormément freiné la fonte du glacier de Sarenne, qui, malgré ce fait, n'a pas encore retrouvé son état de 1975;

- un régime exceptionnel, de $5 \mathrm{~m} 60-1^{\text {et }}$ rang (1,98 d'écart par rapport à la moyenne de 3,62 ).

Les figures 6, 7, 8 et 9 donnent l'évolution pour chacune des 5 stations, des hauteurs de neige (fig. 6) des équivalents en eau (fig. 7) des densités (fig. 8) et des vitesses d'ablation (fig. 9).

\section{Conclusion}

Depuis 1948, première année des relevés de Sarenne, un travail de terrain considérable a été réalisé. Au total, plus de 200 relevés se sont succédés permettant de suivre correctement l'évolution d'un glacier de cirque, glacier se comportant comme un "pluviomètre".

La série de 35 années de mesures sans faille prend un intérêt particulier du fait qu'elle est relativement unique dans l'histoire des relevés glaciologiques. L'utilisation qui en est faite par le CNRS (Louis Raynaud, CNRS glaciologie, Serge Martin, CNRS climatologie) valorise ce travail de terrain et nous incite à le poursuivre. 

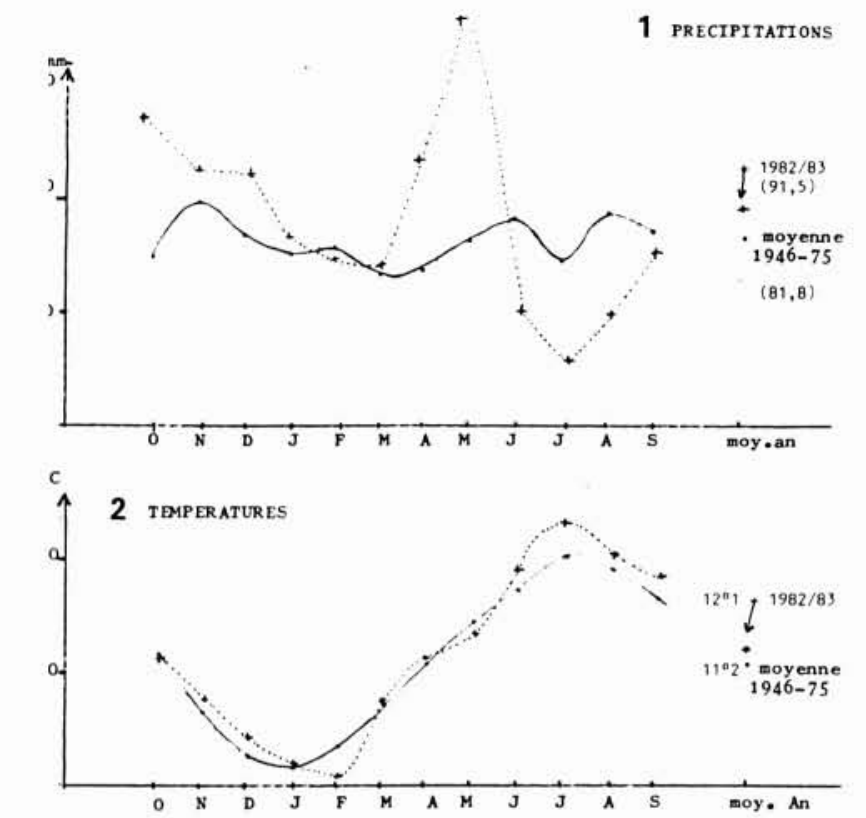

3 DONNEES DE CRENOBLE - SAINI MARIIN D'HERES

\begin{tabular}{|c|c|c|c|c|c|c|c|c|c|c|c|c|c|}
\hline & Oct & Nov & Ded & $\mathrm{Ja}$ & Fev & $\mathrm{Ma}$ & Avr & Mai & Jui & Juil & Aou : & Sep & moy,an \\
\hline $\begin{array}{l}\text { íratures 1982/83 } \\
\text { n degres }\end{array}$ & $11^{\circ} 8$ & $\theta^{\circ}$ & 402 & 207 & $1 \circ 8$ & $7^{\circ} 7$ & $11^{\circ}$ & 1307 & 1907 & $24 \cdot 4$ & $21^{\circ} 2$ & $18^{\circ} 2$ & $12^{\circ}$, \\
\hline $\begin{array}{l}\text { ipitations } 82 / 83 \\
n \text { min }\end{array}$ & 134 & 113 & 112 & 84 & 73 & 76 & 122 & $174 !$ & st & 33 & 49 & 79 & $\begin{array}{l}1098 \mathrm{mr} \\
91.5 \mathrm{moy} \\
\end{array}$ \\
\hline $1946-75$ & 11,8 & 6,5 & 2,5 & 1,9 & 30 & 7,5 & 11. & $14,7 \mid$ & 17,9 & 20,3 & 19,8 & 16,9 & $11^{\circ} 2$ \\
\hline $1946-79$ & 74,8 & 100 & 84,4 & 77,5 & $7 \%$ & 68 & 59,4 & 81,4 & 927 & $73^{2}$ & 94,5 & 86,6 & $\begin{array}{l}981,5 \\
81,5 / \text { move }\end{array}$ \\
\hline
\end{tabular}

\section{M_A_R_C_H_E_DE_L'A_B_L_A T_I_O N}

\begin{tabular}{|l|r|r|r|r|r|}
\hline $\begin{array}{l}\text { Date de 1a } \\
\text { econnaissance }\end{array}$ & Accumulation & $\begin{array}{l}\text { Durée } \\
\text { Période }\end{array}$ & Ab1ation & $\begin{array}{l}\text { Vitesse } \\
\text { ablation }\end{array}$ & $\begin{array}{c}\text { BrLAN } \\
\text { depuis 1er oct. }\end{array}$ \\
\hline juin 1983 & $285 \mathrm{~cm}$ & & & & \\
\hline 8 juin & $181 \mathrm{~cm}$ & 26 & 104 & $4,0 \mathrm{~cm} / \mathrm{s}$ & 181 \\
\hline 6 juillet & $96 \mathrm{~cm}$ & 28 & 85 & $3,0 \mathrm{~cm} / \mathrm{s}$ & 96 \\
\hline 6 aoùt & $66 \mathrm{~cm}$ & 21 & 30 & $1,4 \mathrm{~cm} / \mathrm{s}$ & 66 \\
\hline 0 septembre & $10 \mathrm{~cm}$ & 45 & 56 & $1,2 \mathrm{~cm} / \mathrm{s}$ & +10 \\
\hline & & & & & \\
\hline & & & & & \\
\hline & & & & & \\
\hline
\end{tabular}

5 C_A.R_A_C_TER_ISST_I_Q_U_ES_DU_C_Y_C_L_E

en millions de $\mathrm{m} 3$ par $\mathrm{km} 2$

et CLASSEMENT par rapport aux cycles mesurés

\begin{tabular}{l|c|c|c|c|c|}
\hline & Valeur & Rang & Moyenne & Ecart type & $\begin{array}{c}\text { cart par rap. } \\
\text { moyenne }\end{array}$ \\
\hline cumulation & 285 & ler & 158 & 0,59 & +127 \\
\hline 1ation & 275 & 4ème & 206 & 0,67 & +69 \\
\hline 1an & +10 & $10 \mathrm{eme}$ & $-0,48$ & 0,86 & +58 \\
\hline gime & 560 & 1er & 362 & 0,94 & +198 \\
\hline
\end{tabular}

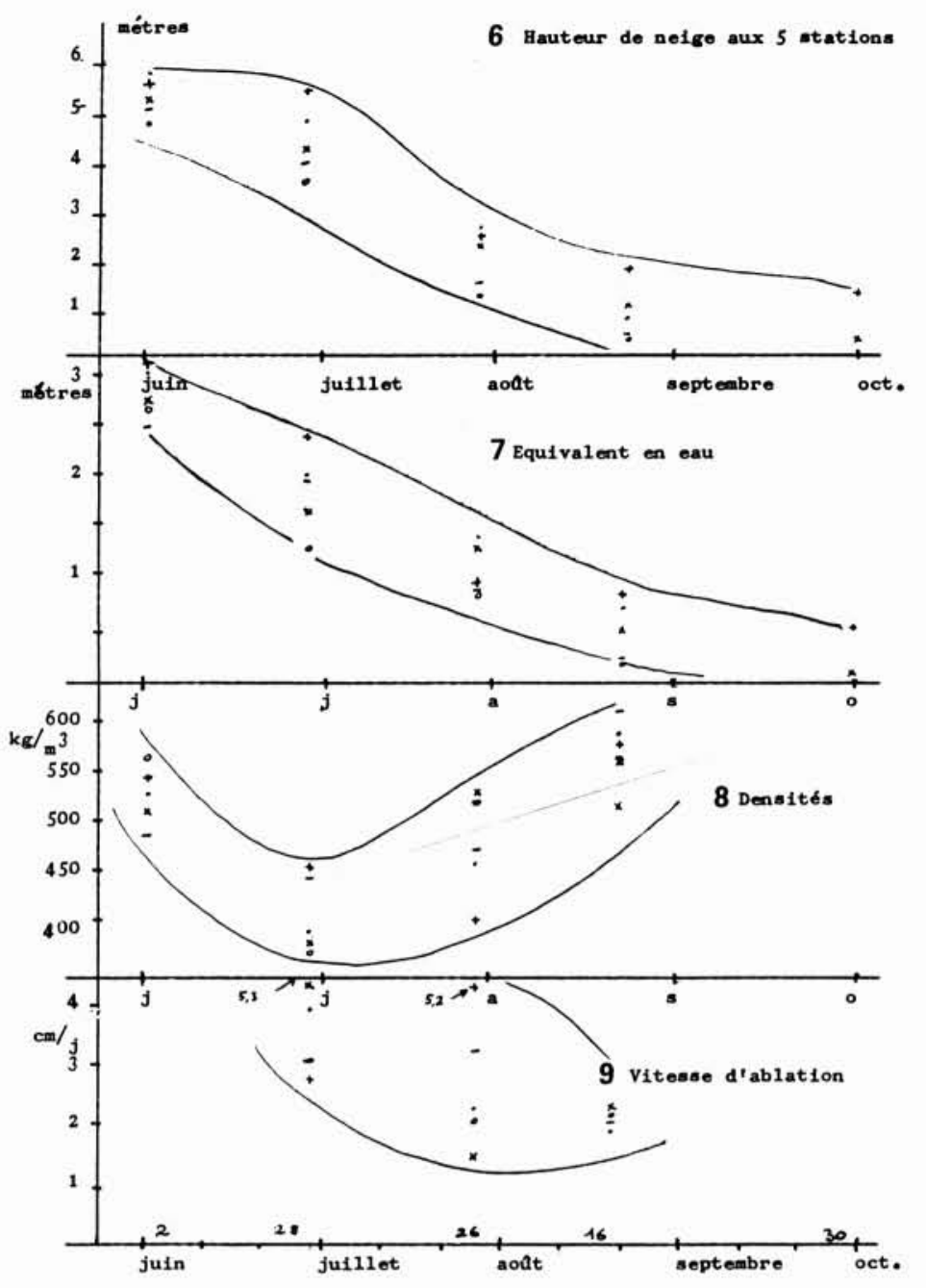

10 caracteristiques des 33 crcles 1948-1983

\begin{tabular}{|c|c|c|c|c|c|}
\hline $\mathrm{N}^{0}$ & ANNEE & ACCUMULATION & ABLAIION & BILAN & REGIME \\
\hline 1 & $1948-49$ & 0,71 & 3,70 & $-2,99$ & 4,41 \\
\hline 2 & $49-50$ & 1,68 & 3,30 & $-1,62$ & 4,98 \\
\hline 3 & $1950-51$ & 2,50 & 2,30 & $+0,20$ & 4,80 \\
\hline 4 & $51-52$ & 1,75 & 3,37 & $-1,62$ & 5,12 \\
\hline 5 & $52-53$ & 1.77 & 2,41 & $-0,64$ & 4,18 \\
\hline 6 & $53-54$ & 1,05 & 1,60 & $-0,55$ & 2,65 \\
\hline 7 & $54-55$ & 2,20 & 1,54 & $+0,66$ & 3,74 \\
\hline 8 & $55-56$ & 1,08 & 1,68 & $-0,60$ & 2,76 \\
\hline 9 & $56-57$ & 1,38 & 1,90 & $-0,52$ & 3,28 \\
\hline 10 & $57-58$ & 1.47 & 2.14 & $-0,67$ & 3,61 \\
\hline 11 & $58-59$ & 1,43 & 2,69 & $-1,26$ & 4,12 \\
\hline 12 & $59-60$ & 1,20 & 1,08 & $+0,12$ & 2,28 \\
\hline 13 & $1960-61$ & 2,15 & 2.54 & $-0,39$ & 4,69 \\
\hline 14 & $61-62$ & 1,55 & 2.46 & $-0,91$ & 4,02 \\
\hline is & $62-63$ & 1,45 & 1,26 & $+0,19$ & 2,72 \\
\hline 16 & $63-64$ & 0,89 & 2.72 & $-1,82$ & 3,61 \\
\hline 17 & $64-65$ & 1,03 & 1,00 & $+0,03$ & 2,03 \\
\hline 18 & $65-66$ & 2,14 & 1,72 & $\cdot 0,42$ & 3,86 \\
\hline 19 & $66-67$ & 1,63 & 2,04 & $-0,41$ & 3,67 \\
\hline 20 & $67-68$ & 1,13 & 0,79 & $+0,34$ & 1,92 \\
\hline 21 & $68-69$ & 1,59 & 1,95 & $-0,36$ & 3,54 \\
\hline 22 & $69-70$ & 1,82 & 2,23 & $-0,41$ & 4,05 \\
\hline 23 & $1970-71$ & 1,22 & 2,32 & $-1,10$ & 3,54 \\
\hline 24 & $71-72$ & 1,01 & 1,38 & $-0,37$ & 2,39 \\
\hline 25 & $72-73$ & 0,95 & 1,82 & $-0,87$ & 2,27 \\
\hline 26 & $73-74$ & 0,88 & 2,48 & $-1,60$ & 3,36 \\
\hline 27 & $74-75$ & 1,32 & 1,21 & $+0,11$ & 2,53 \\
\hline 28 & $75-76$ & 0,33 & 2,40 & $-2,07$ & 2,73 \\
\hline 29 & $76-77$ & 2,78 & 1,79 & $+0,99$ & 4,57 \\
\hline 30 & $77-78$ & 2,07 & 1,52 & $+0,55$ & 3.59 \\
\hline 31 & $78-79$ & 2.26 & 2,37 & $-0,11$ & 4,63 \\
\hline 32 & $79-80$ & 2.25 & 1,93 & $+0,32$ & 4,18 \\
\hline 33 & $1980-81$ & 1,65 & 1,61 & $+0,04$ & 3,26 \\
\hline 34 & $81-82$ & 2,03 & 2,13 & $-0,10$ & 4,16 \\
\hline 35 & $82-83$ & 2,85 & 2,75 & $+0,10$ & 5,60 \\
\hline
\end{tabular}




\section{Exposé de M. REYNAUD}

M. Le Président fait remarquer que l'avance des glaciers est bien coûteuse pour l'électricien qui préfèrerait un front de glacier stable et invite l'auditoire à questionner M. ReYnaud.

Contrairement à l'ancienne hypothèse, rappelée par M. BEZINGE, selon laquelle les glaciers auraient un temps de retard par rapport aux variations climatiques, dépendant de leur situation géographique vis-à-vis de l'influence atlantique ou méditerranéenne, M. REYNAUD penche plutôt pour une certaine unité dans le comportement des glaciers alpins, le facteur déterminant étant, selon lui, la longueur et non pas la situation géographique.

Répondant à une question de $M$. BEZINGE sur la disparition de l'eau au glacier d'Argentière, M. BERnARD pense que l'obstruction des captages est due principalement à l'augmentation de lépaisseur de la glace, créant une compression capable d'obstruer les conduits amenant l'eau.

M. GAUTHERON précise que la transition observée n'a pas été brutale, on peut distinguer deux périodes : avant 1968, où le passage de l'eau se faisait au point bas situé en rive gauche; après 1978, où il a été constaté qu'il se faisait constamment en rive droite. La transition apparente a duré une dizaine d'années, la transition réelle au moins une dizaine d'années.

$M$. BEZINGE confirme ces analyses en insistant sur le rôle de la pression hydrostatique de la glace dans le changement des écoulements sous-glaciaires.

M. GAUTHERon évoque aussi la possibilité d'effets de seuil; à ce sujet, M. ReYnaud cite l'exemple du glacier Blanc, des photographies de 1933-36 montrent une ressemblance certaine avec ce qui s'est passé plus récemment à Argentière.

M. ROBERT revient sur le problème de l'écoulement de l'eau à l'intérieur du glacier, les documents de M. BEZINGE montrent bien, selon lui, l'importance des efforts à l'intérieur du glacier; à n'en pas douter, ils doivent provoquer des modifications importantes et imprévisibles dans le cheminement de l'eau. M. Le Président regrette que l'on ne puisse avoir plus de certitude ou d'explication rigoureuse sur ce sujet.
M. de CRECY intervient pour rendre compte des renseignements donnés par MM. AELLEN et KASSER sur l'avance des glaciers suisses; ils confirment les phénomènes observés en France par MM. Valla et ReYNAUd : bilan au voisinage de zéro, mais remarquent une certaine tendance au déficit en versant Nord, et des bilans nuls ou même positifs en versant Sud. A ce propos, $M$. Le Président demande à M. BEZINGE ce qu'il pense des records de précipitations signalés sur le Tessin. M. BEZINGE ne peut conclure qu'au caractère exceptionnel de la région et des phénomènes observés : précipitations avec des intensités record pour l'Europe.

Exposé de M. VALLA

Revenant sur la corrélation température-précipitation, $M$. Le Président demande au conférencier quel est le facteur déterminant dans l'étude du bilan. Les deux facteurs interviennent, répond M. VALLA, mais différemment selon la période : les précipitations sont en relation directe avec l'accumulation observée en juin, les températures interviennent de manière prépondérante sur la fonte pendant les mois d'été; il y a des exceptions remarquables : une chute de neige en août modifie lalbedo et réfléchit l'énergie solaire, elle perturbe alors sensiblement le bilan de fonte.

$M$. Le Président fait remarquer que nous venons de subir une période exceptionnelle avec sept années consécutives d'hydraulicité positive, à opposer aux cinq années d'hydraulicité négative autour de 1960; il est ainsi possible, selon lui, de lier cette hydraulicité à l'avance ou au recul des glaciers observés aux mêmes époques. M. ReYNAUD reviendra sur ce point dans son exposé.

Répondant à M. LÉvY, M. VALLA confirme que la précision obtenue sur le bilan est de l'ordre de $15-20 \mathrm{~cm}$ de valeur en eau. Rassurant M. POIREL, il nous assure que l'ablation est négligeable en automne : la surface du glacier reste continuellement glacée dès fin septembre, et les carottages effectués à la même époque ont montré le peu d'importance du phénomène. 